\title{
Association of continuity of care with blood pressure control in patients with chronic kidney disease and hypertension
}

\begin{abstract}
Masuma A Khanam, Alex Kitsos, Jim Stankovich, Ronald Castelino, Matthew Jose, Gregory M Peterson, Barbara Wimmer, Tabish Razi Zaidi, Jan Radford
\end{abstract}

\section{Background and objective}

Hypertension frequently co-exists with chronic kidney disease (CKD). The objective of this study was to investigate blood pressure (BP) control among general practice patients with CKD and hypertension, and whether control was related to continuity of care.

\section{Methods}

We analysed data from NPS MedicineWise Medicinelnsight, examining the achievement of guideline-recommended BP levels in patients with CKD and hypertension, and the relationship with sociodemographic, clinical and healthsystem variables, including continuity of care $(\mathrm{CoC})$ in general practice.

\section{Results}

Of 37,425 patients in the cohort, $46.7 \%$ had achieved the recommended BP targets. Patients with higher relational $\mathrm{CoC}$ and more general practitioner (GP) visits were more likely to achieve $\mathrm{BP}$ targets, while this was less likely when the target was lowered by concomitant diabetes or cardiovascular disease.

\section{Discussion}

Reaching BP targets in patients with CKD is a challenge, especially when the target is lower because of co-existing risk factors. Greater $\mathrm{CoC}$ from the same GP increased the likelihood of achieving target $\mathrm{BP}$ values.
CHRONIC KIDNEY DISEASE (CKD) affects approximately $10 \%$ of adult Australians. ${ }^{1}$ CKD is associated with significantly increased risk of cardiovascular disease (CVD) and cardiovascular (CV) death. ${ }^{2}$ The risk of dying from CVD is up to 20 times greater in patients with CKD than the risk of requiring dialysis or transplantation. ${ }^{3}$

Hypertension frequently co-exists with $\mathrm{CKD} ;{ }^{4}$ its prevalence rises with advancing CKD stage, alongside blood pressure (BP) becoming more difficult to control. ${ }^{5}$ Hypertension is a common cause of end-stage renal disease (ESRD) and is associated with more rapid progression of CKD. ${ }^{2,6,7}$ Hypertension is therefore an important therapeutic target in the management of CKD; lowering elevated $\mathrm{BP}$ is an effective strategy to both reduce the risk of $\mathrm{CV}$ events and slow progression of CKD. ${ }^{8-10}$ Kidney Health Australia ${ }^{11}$ and the National Heart Foundation of Australia ${ }^{12}$ provide guidelines to manage $\mathrm{BP}$ and indicate target levels in patients with CKD, according to the presence or absence of specific high-risk clinical factors $(\leq 130 / 80 \mathrm{mmHg}$ for patients with concomitant diabetes, CVD or albuminuria; $\leq 140 / 90 \mathrm{mmHg}$ for others).

Factors contributing towards BP control relate to patients, doctors and health systems. ${ }^{13}$ Continuity of care (CoC) is a widely accepted core principle of primary care, ${ }^{14}$ and is an important component of high-quality healthcare as it spans all the factors contributing to BP control. Better awareness of hypertension and adherence to medication by patients has been reported with higher $\mathrm{CoC} ;{ }^{15}$ it has also been shown to result in reduced hospitalisation risks, emergency department visits and healthcare costs for several chronic diseases. ${ }^{16}$ CKD and hypertension are common chronic diseases and are routinely managed in primary care. ${ }^{17,18}$ Hence, it is worth looking at the association of $\mathrm{CoC}$ with BP control in Australian general practice patients with CKD and concomitant hypertension. The aim of this study was therefore to investigate the control of BP, according to guidelinerecommended targets, in adults with CKD and hypertension, and to evaluate factors related to BP control, particularly continuity of general practice care.

\section{Methods}

MedicineInsight, developed and managed by NPS MedicineWise, is a large-scale national data program in Australia to extract and collate longitudinal, whole-of-practice data from the clinical information systems of consenting general practices. MedicineInsight collects de-identified patient data, including demographics, encounters (not including progress notes), diagnoses, prescriptions and pathology tests. At July 2017, MedicineInsight had recruited over 650 general practices, giving information from more than 3300 general practitioners (GPs) and 3.6 million patients. ${ }^{19}$ 


\section{Participants}

We used MedicineInsight data from 1 January 2013 to 1 June 2016 collected from 329 general practices located throughout Australia. Patients were initially included if at the time of data extract they were aged at least 18 years, met The Royal Australian College of General Practitioners' (RACGP's) definition of an 'active' patient (at least three consultations with a GP at the same general practice within a two-year period) and could be diagnosed with CKD by a GP on the basis of their laboratory pathology results (having two or more estimated globular filtration rate $\left[\mathrm{eGFR}\right.$ ] values $<60 \mathrm{~mL} / \mathrm{min} / 1.73 \mathrm{~m}^{2}$, and/or two urinary albumin-to-creatinine ratios (ACRs) $\geq 3.5 \mathrm{mg} / \mathrm{mmol}$ in females or $\geq 2.5 \mathrm{mg} / \mathrm{mmol}$ in males, at least 90 days apart). ${ }^{11}$

\section{Variables}

We considered age group, sex, rurality, Aboriginal and/or Torres Strait Islander status and socioeconomic index as sociodemographic indicators, and ACR and eGFR as laboratory variables. Rurality was based on Australian Bureau of Statistics' Australian Statistical Geography Standard Remoteness Areas ${ }^{20}$ and assigned according to the postcode of the patient's residence. The remoteness classification divides each state and territory into several regions on the basis of their relative access to services. Socioeconomic index was also based on patient postcode, using the Socio-Economic Indexes for Areas (SEIFA) of relative socioeconomic advantage and disadvantage decile. SEIFA is ranked from 1 (most disadvantaged area) to 10 (most advantaged). ${ }^{21}$

We also analysed each patient's comorbidities, documentation of a diagnosis of CKD, number of visits with a GP and their relational $\mathrm{CoC}$ with a particular GP. Documentation of the diagnosis of CKD was extracted from condition codes and manual searches (including terms such as 'chronic renal disease' and 'chronic renal impairment') conducted on 'free-text' or narrative information in the past medical history, reason for encounter and reason for prescription data fields. The comorbidities examined were based on 'condition flags' provided by MedicineInsight, using an algorithm that analyses coded and free-text patient information. We examined only hypertension, diabetes, CVD, anxiety and atrial fibrillation as comorbidities, as these each had a prevalence above $10 \%$. The diagnoses of CVD (myocardial infarction, heart failure, coronary artery bypass graft, transient ischaemic attack and stroke) were provided by MedicineInsight using methods described in their data book. ${ }^{22}$

A total of 61,102 patients had laboratory evidence of CKD within the study period. We identified 51,242 (83.9\%) of these patients with an additional diagnosis of hypertension. The final study population consisted of $37,425(73.0 \%)$ of these patients who had recorded BP measurements available during the period of between six and 12 months following the time of laboratory evidence of CKD. This time period was used to allow for delays in any changes to therapy and the gradual achievement of the desired BP level. The BP used in the analyses was the last recorded in this period for each patient. If two BP measurements were registered on any single date, the lowest measurement was used. Target BP values were taken as $\leq 130 / 80 \mathrm{mmHg}$ for patients with concomitant diabetes, CVD or albuminuria, and $\leq 140 / 90 \mathrm{mmHg}$ for the remainder. ${ }^{11}$

To recap, the final set of 37,425 patients were (i) active (at least three GP visits within two years at any point within the whole data collection period of three years and five months); (ii) had laboratory evidence of CKD within the data collection period, with an additional diagnosis of hypertension; and (iii) had recorded BP measurements available during the period between six and 12 months following the time of laboratory evidence of CKD. CoC was then calculated for each included patient after the time of laboratory evidence of CKD, over the remainder of the data collection period, using the HerfindahlHirschman Index, which has been shown to be highly correlated with other common measures of CoC. ${ }^{23}$ Low CoC in general practice, measured with this index, has also been associated with a higher risk of mortality. ${ }^{24,25}$ If $p_{i}$ is the proportion of a patient's visits where the patient sees doctor $i, \mathrm{CoC}=\sum p_{i}{ }^{2}$.

For example, if a patient makes 10 visits to GPs - seven visits (70\%) to doctor A, two visits (20\%) to doctor $\mathrm{B}$, and 1 visit (10\%) to doctor $\mathrm{C}$ - the patient's $\mathrm{CoC}$ is $0.7^{2}+$ $0.2^{2}+0.1^{2}=0.54$ (medium). If another patient also makes 10 visits to GPs, but four of them to doctor $A$, three of them to doctor $\mathrm{B}$, and three to doctor $\mathrm{C}, \mathrm{CoC}$ is lower $\left(0.4^{2}+0.3^{2}+0.3^{2}=0.34\right.$; low $)$. CoC can take values ranging from close to 0 (a large number of visits, all to different doctors) to 1 (all visits to the same doctor). Patients were subsequently categorised into four groups on the basis of the $\mathrm{CoC}$ - those with maximum perfect continuity and the remainder split by approximate tertiles (cut points of 0.5 and 0.75 ).

\section{Data analysis}

Statistical calculations were performed using the language of R. ${ }^{26}$ Multivariable logistic regression analysis was used to identify factors associated with achievement of recommended target BP levels. Variables identified as being associated with achieving adequate BP control through univariate analysis, as well as age group, sex, baseline CKD stage, rurality, socioeconomic status and Aboriginal and/or Torres Strait Islander status, were all initially included in the regression model. A backwards selection procedure was implemented to determine the final regression model that included the review of nested models and analysis of likelihood ratio tests, and residual plots. Consequently, the number of GP encounters, GPs' documentation of CKD, CoC and presence of CVD and diabetes were also included in the final regression model. As a result of the large sample size, patients and measurements, a two-sided $P$ value of $<0.001$ was deemed to be statistically significant for the logistic regression model. The Tasmanian Health and Medical Human Research Ethics Committee approved the study (H0015651). 


\section{Results}

Characteristics of the patients are shown in Table 1 . There was a predominance of patients aged $\geq 70$ years $(81.7 \%)$. Figure 1 shows the distribution of $\mathrm{CoC}$ in the sample population. Overall, 17,469 (46.7\%) of the patients had achieved a target BP; this proportion was $61.5 \%$ among the patients without concomitant diabetes, CVD or albuminuria, and only $38.2 \%$ in those with a lower target because of the presence of at least one of these factors.

Figure 2 shows the concentration of patients with specific BP levels by different cut-offs, in scatter plots. It was mostly the systolic BP that was higher than recommended among the patients without concomitant diabetes, CVD or albuminuria (high density of points within the lower right-hand quadrant of Figure 2), while both diastolic and systolic $\mathrm{BP}$ were often higher than the target level in individuals with one or more of these factors (high density of points within both the upper and lower right-hand quadrants).

Table 2 presents results from the final multivariable logistic regression model, showing factors independently associated with BP control. Higher CoC was positively associated with $\mathrm{BP}$ control; seeing a single GP increased the likelihood of having a BP below the target level by $22 \%$, after adjusting for all other variables. Similarly, seeing a GP more frequently (a doubling in number of GP visits within the period of time after laboratory evidence of CKD and the remainder of the data collection period) increased the odds of having a $\mathrm{BP}$ below the target level by $18 \%$. The mean (standard deviation) number of GP visits within the 12 months following laboratory evidence of CKD was 15.5 (10.9). CoC tended to be higher within major cities and in the most advantaged; however, $\mathrm{CoC}$ was a stronger independent predictor of $\mathrm{BP}$ control than either rurality or socioeconomic index.

Patients with coexisting diabetes or CVD, and therefore having lower BP targets, were far less likely to have their BP below the target level. The independent effects of age and sex were much smaller, with older age groups (80-89 years and $\geq 90$ years) and females less likely to have achieved a target BP.

\section{Discussion}

Less than half of the patients with CKD and hypertension had BP values meeting the guideline-recommended target values. This was predominantly due to individuals not achieving the stricter BP goal $(\leq 130 / 80 \mathrm{mmHg}$ ) recommended if there was concomitant diabetes, CVD and/ or albuminuria. These results probably reflect the difficulties in achieving a lower target BP in a largely older group of patients, also at risk of hypotension and falls. Although evidence suggests patients aged $\geq 75$ years gain an overall benefit from lowering BP, ${ }^{27}$ many GPs may be reluctant to aim for BPs $\leq 140 / 90 \mathrm{mmHg}$ or $\leq 130 / 80 \mathrm{mmHg}$, fearing the patient's falls risk outweighs other health gains. ${ }^{27}$ We did not, however, attempt to examine hypotension in the MedicineInsight data. GPs may also be reluctant to aggressively lower BP as there is uncertainty about the effect of intensive BP-lowering on renal function in these older patients. ${ }^{28}$ Further research is required to directly examine this particular area.

There is scarcity of data on BP control in patients with CKD in Australia. One study found that among patients with CKD who were prescribed BP-lowering agents, 61\% did not meet the guideline-recommended BP target. ${ }^{29}$ Apart from avoiding the dangers of inducing hypotension in the elderly, possible reasons for the observed practice-guideline gap include resistant hypertension (especially with concomitant CKD), poor adherence with therapy and inadequate use of antihypertensive drugs..$^{8,30,31}$

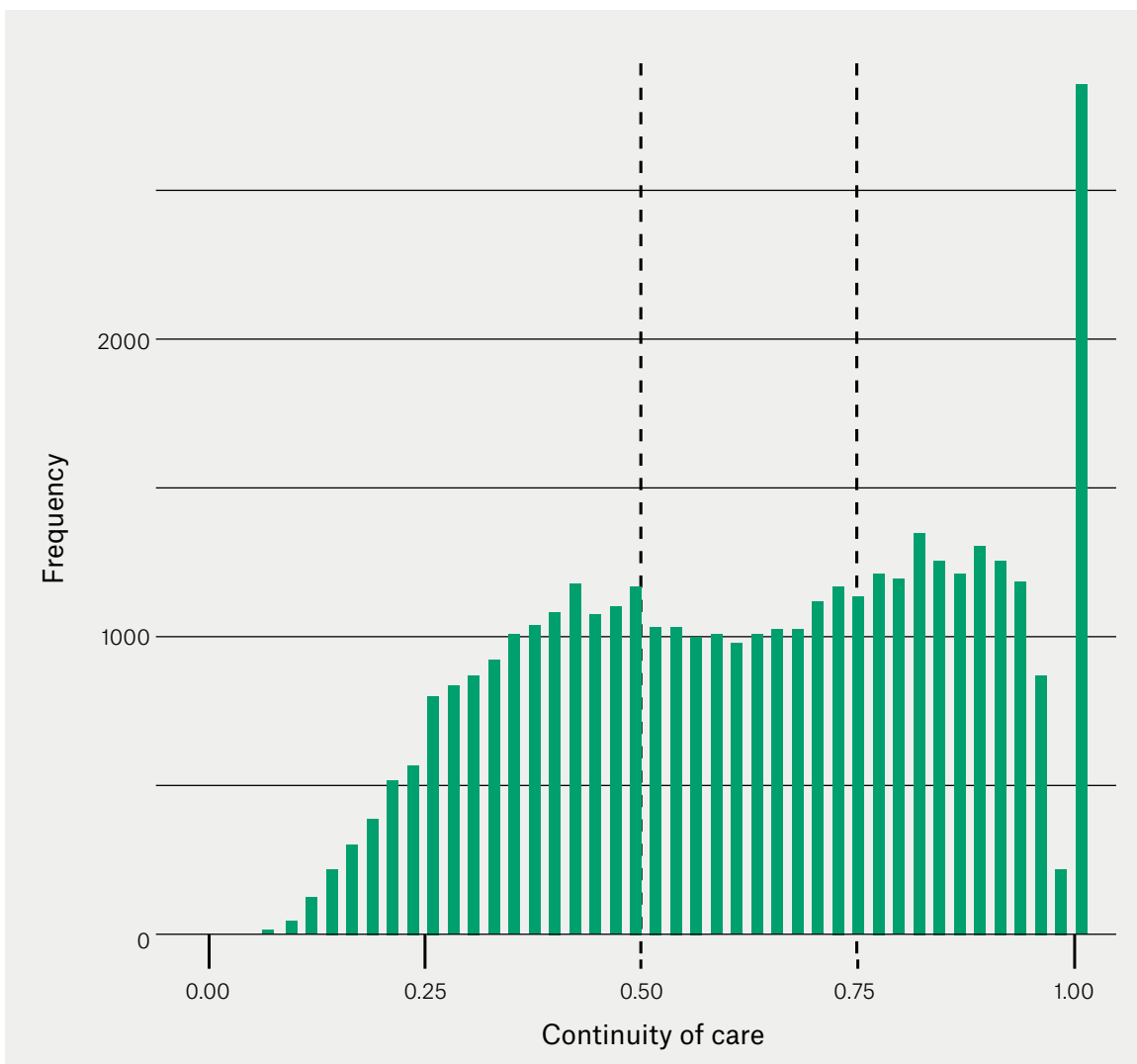

Figure 1. Distribution of continuity of care among the patients with chronic kidney disease and hypertension 
Importantly, patients who had more visits to a GP and who had higher relational CoC were more likely to achieve target BP levels. A dose-response relationship was found, with patients with the maximum $\mathrm{CoC}$ (care from the same GP for the analysis period) being $22 \%$ more likely to have achieved the recommended target BP levels. Thus, continuity of general practice care - the ongoing therapeutic relation between the patient and a single GP that bridges episodes of care ${ }^{32,33}$ - has seemingly exerted an impact in this study on achieving target BP levels. To the best of our knowledge, our study is the first in Australia to look at the association of $\mathrm{CoC}$ with achievement of guidelinerecommended BP control among patients with CKD.

Patients with target blood pressure of $140 / 90 \mathrm{mmHg}(\mathrm{n}=13,220)$

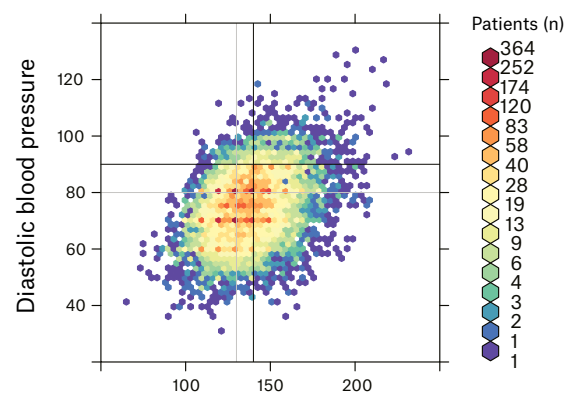

Systolic blood pressure

Patients with target blood pressure of $130 / 80 \mathrm{mmHg}(n=24,205)$

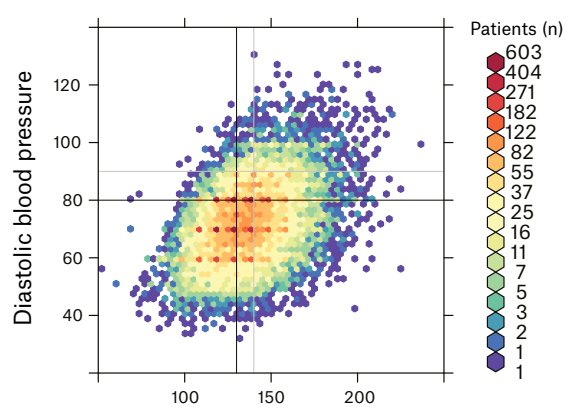

Systolic blood pressure

Figure 2. Distribution of the patients with chronic kidney disease and hypertension, by different blood pressure targets
This study comprised a large cohort of patients from general practices across the country. The patient characteristics of the dataset were similar to the Australian population. Despite the strengths, this study has some limitations. The cross-sectional nature of the study could only retrospectively investigate the association of the $\mathrm{CoC}$ with BP control, and not determine causation. Each participating general practice has chosen to be part of the MedicineInsight program. Patient information was collected by GPs for clinical decision-making, not research purposes. All health and demographic information may not have been entered in the medical record system. For example, Aboriginal and Torres Strait Islander status was missing for approximately $22 \%$ of the patients. It is possible that $\mathrm{BP}$ values were recorded within patient notes and not included in the research data.

The recording of clinical encounters or visits, used to calculate $\mathrm{CoC}$, may have limitations. In general practice information systems, an encounter is recorded any time a patient's electronic medical record is accessed, regardless of whether this was for clinical purposes. MedicineInsight has a set of criteria to probabilistically define a clinical encounter and exclude administrative encounters. These include being associated with a GP or practice nurse, not including terms in 'reason for encounter' that indicate an administrative record only, containing at least one entry in the 'diagnosis', 'reason for encounter' or 'reason for prescription' fields, and having a duration longer than three minutes. However, it is possible that some administrative encounters were still included in our analysis. Finally, tracking patients' attendance at multiple practices was not possible.

In conclusion, attainment of the guideline-recommended BP levels was reasonably good in patients with $\mathrm{CKD}$ and hypertension, without other risk factors. However, it was much poorer in the presence of concomitant diabetes or CVD, seemingly due to a lower BP target. The study also highlighted the importance of more GP visits, and especially the CoC, in the management of hypertension in patients with CKD. Based on the results, it would seem beneficial for GPs to increase the $\mathrm{CoC}$ given to patients with CKD, with special attention given to females and older individuals.

\section{Authors}

Masuma A Khanam MBBS, MPH, PhD, Postdoctoral Research Fellow, School of Health Sciences, University of Tasmania, Hobart, Tas. masuma.khanam@utas.edu.au

Alex Kitsos BPhty (Hons), MMedStat, Health Analyst College of Health and Medicine, University of Tasmania, Hobart, Tas

Jim Stankovich PhD, Statistician, Monash University, Melbourne, Vic; Health Services Innovation Tasmania, School of Medicine, University of Tasmania, Hobart, Tas

Ronald Castelino BPharm, MPharm, PhD, Lecturer in Pharmacy, University of Sydney, Sydney, NSW: Adjunct Lecturer, University of Tasmania, Hobart, Tas Matthew Jose MBBS, FRACP, PhD, FASN, AFRACMA, Professor of Medicine, University of Tasmania, Hobart, Tas; Consultant Nephrologist, Royal Hobart Hospital, Hobart, Tas

Gregory M Peterson BPharm, PhD, MBA, FSHP, FACP, GAICD, AACPA, ARPharmS, MPS, Professor of Pharmacy and Director of Health Services Innovation Tasmania, School of Medicine, University of Tasmania, Hobart, Tas

Barbara Wimmer BPharm, MSc, PhD, Lecturer of Pharmacy, College of Health and Medicine, University of Tasmania, Hobart,Tas

Tabish Razi Zaidi BPharm, MPharm, PhD, Lecturer of Pharmacy, College of Health and Medicine, University of Tasmania, Hobart, Tas

Jan Radford MBBS, FRACGP, RACGP, M Psych Med, MEd, FARGP, Associate Professor of General Practice, Launceston Clinical School, University of Tasmania, Launceston, Tas

Competing interests: None.

Funding: None.

Provenance and peer review: Not commissioned, externally peer reviewed.

\section{Acknowledgement}

The authors would like to acknowledge NPS MedicineWise for providing the data.

\section{References}

1. Australian Institute for Health and Welfare. Chronic kidney disease. Canberra, ACT: AlHW, 2015. Available at www.aihw.gov.au/chronickidney-disease [Accessed 23 October 2018].

2. Go AS, Chertow GM, Fan D, McCulloch CE, Hsu CY. Chronic kidney disease and the risks of death, cardiovascular events, and hospitalization. N Engl J Med 2004;351(13):1296-305. doi: 10.1056/NEJMoa041031.

3. Van Gelder VA, Scherpbier-De Haan ND, De Grauw WJ, et al. Quality of chronic kidney disease management in primary care: A retrospective study. Scand J Prim Health Care 2016;34(1):73-80. doi: 10.3109/02813432.2015.1132885.

4. Rao MV, Qiu Y, Wang C, Bakris G. Hypertension and CKD: Kidney Early Evaluation Program (KEEP) and National Health and Nutrition Examination Survey (NHANES), 1999-2004. Am J Kidney Dis 2008;51(4 Suppl 2):S30-37. doi: 10.1053/j. ajkd.2007.12.012. 


\section{Table 1. Characteristics of patients with chronic kidney disease and hypertension, by sex}

\begin{tabular}{|c|c|c|c|c|}
\hline Variable & n (\%) & $\begin{array}{r}\text { Female } \\
21,071(56.3)\end{array}$ & $\begin{array}{r}\text { Male } \\
16,354(43.7)\end{array}$ & $P$ value \\
\hline \multicolumn{5}{|l|}{ Age group, years } \\
\hline$<70$ & $6,872(18.6)$ & $3,358(15.9)$ & $3,514(21.5)$ & $<0.001$ \\
\hline $70-79$ & 13,232 (35.4) & 7,057 (33.5) & $6,175(37.8)$ & \\
\hline 80-89 & 14,188 (37.9) & $8,526(40.5)$ & $5,662(34.6)$ & \\
\hline$\geq 90$ & $3,133(8.4)$ & $2,130(10.1)$ & $1,003(6.1)$ & \\
\hline \multicolumn{5}{|l|}{ CKD stage } \\
\hline $3 a$ & $25,107(67.1)$ & $14,130(67.1)$ & $10,977(67.1)$ & $<0.001$ \\
\hline $3 b$ & $9,599(25.7)$ & $5,498(26.1)$ & $4,101(25.1)$ & \\
\hline 4 & $2,358(6.3)$ & $1,283(6.1)$ & 1,075 (6.6) & \\
\hline 5 & $361(1.0)$ & $160(0.8)$ & $201(1.2)$ & \\
\hline \multicolumn{5}{|l|}{ Diabetes } \\
\hline Yes & 13,044 (34.9) & 6,664 (31.6) & $6,380(39.0)$ & $<0.001$ \\
\hline \multicolumn{5}{|l|}{ CVD } \\
\hline Yes & $15,640(41.8)$ & 7,901 (37.5) & 7,739 (47.3) & $<0.001$ \\
\hline \multicolumn{5}{|l|}{ Atrial fibrillation } \\
\hline Yes & $6,014(16.1)$ & $3,203(15.2)$ & $2,811(17.2)$ & $<0.001$ \\
\hline \multicolumn{5}{|l|}{ Anxiety } \\
\hline Yes & $5,122(13.7)$ & $3,655(17.3)$ & $1,467(9.0)$ & $<0.001$ \\
\hline \multicolumn{5}{|l|}{ Rurality } \\
\hline Major cities & 21,926 (58.6) & $12,500(59.6)$ & $9,426(57.9)$ & $<0.001$ \\
\hline Inner regional & $10,080(26.9)$ & $5,661(27.0)$ & $4,419(27.2)$ & \\
\hline Outer regional & $4,781(12.8)$ & $2,583(12.3)$ & $2,198(13.5)$ & \\
\hline Remote & $474(1.3)$ & $246(1.2)$ & $228(1.4)$ & \\
\hline \multicolumn{5}{|l|}{ SEIFA } \\
\hline Most disadvantaged & $10,863(29.0)$ & $6,099(29.1)$ & $4,764(29.3)$ & 0.760 \\
\hline Middle & $15,551(41.6)$ & $8,795(41.9)$ & $6,756(41.6)$ & \\
\hline Most advantaged & $10,818(28.9)$ & $6,081(29.0)$ & $4,737(29.1)$ & \\
\hline \multicolumn{5}{|l|}{ Indigenous status* } \\
\hline Aboriginal/Torres Strait Islander & $400(1.4)$ & $229(1.4)$ & $171(1.3)$ & 0.711 \\
\hline Non-Indigenous & $28,745(98.6)$ & $16,154(98.6)$ & $12,591(98.7)$ & \\
\hline \multicolumn{5}{|l|}{ GP-documented CKD } \\
\hline Yes & $9,480(25.3)$ & $5,013(23.8)$ & $4,467(27.3)$ & $<0.001$ \\
\hline \multicolumn{5}{|l|}{ GP continuity of care } \\
\hline Low & 12,406 (33.2) & $7,074(33.6)$ & $5,332(32.6)$ & $<0.001$ \\
\hline Middle & $10,998(29.4)$ & $6,315(30.0)$ & $4,683(28.6)$ & \\
\hline High & $11,170(29.9)$ & $6,204(29.4)$ & $4,966(30.4)$ & \\
\hline Maximum (1) & $2,849(7.6)$ & $1,476(7.0)$ & $1,373(8.4)$ & \\
\hline
\end{tabular}

*Data missing for $22 \%$ of the sample

CKD, chronic kidney disease; CVD, cardiovascular disease; GP, general practitioner; SEIFA, Socio-Economic Indexes for Areas

5. Cai G, Zheng Y, Sun X, Chen X; Survey of Prevalence, Awareness, and Treatment Rates in Chronic Kidney Disease Patients with Hypertension in China Collaborative Group. Prevalence, awareness, treatment, and control of hypertension in elderly adults with chronic kidney disease: Results from the survey of Prevalence, Awareness, and Treatment Rates in Chronic Kidney Disease Patients with Hypertension in China. J Am Geriatr Soc 2013;61(12):2160-67.
6. Botdorf J, Chaudhary K, Whaley-Connell A Hypertension in cardiovascular and kidney disease. Cardiorenal Med 2011;1(3):183-92. doi: 10.1159/000329927.

7. Segura J, Ruilope LM. Hypertension in moderate-to-severe nondiabetic CKD patients. Adv Chronic Kidney Dis 2011;18(1):23-27. doi: 10.1053/j. ackd.2010.11.001.
8. de Galan BE, Perkovic V, Ninomiya T, et al. Lowering blood pressure reduces renal events in type 2 diabetes. J Am Soc Nephrol 2009;20(4):883-92. doi: 10.1681/ASN.2008070667.

9. Jafar $\mathrm{TH}$, Stark PC, Schmid $\mathrm{CH}$, et al. Progression of chronic kidney disease: The role of blood pressure control, proteinuria, and angiotensin-converting enzyme inhibition: A patient-level meta-analysis. Ann Intern Med 2003:139(4):244-52. 
Table 2. Multivariable logistic regression: Factors associated with achieving specific BP targets in patients with chronic kidney disease and hypertension

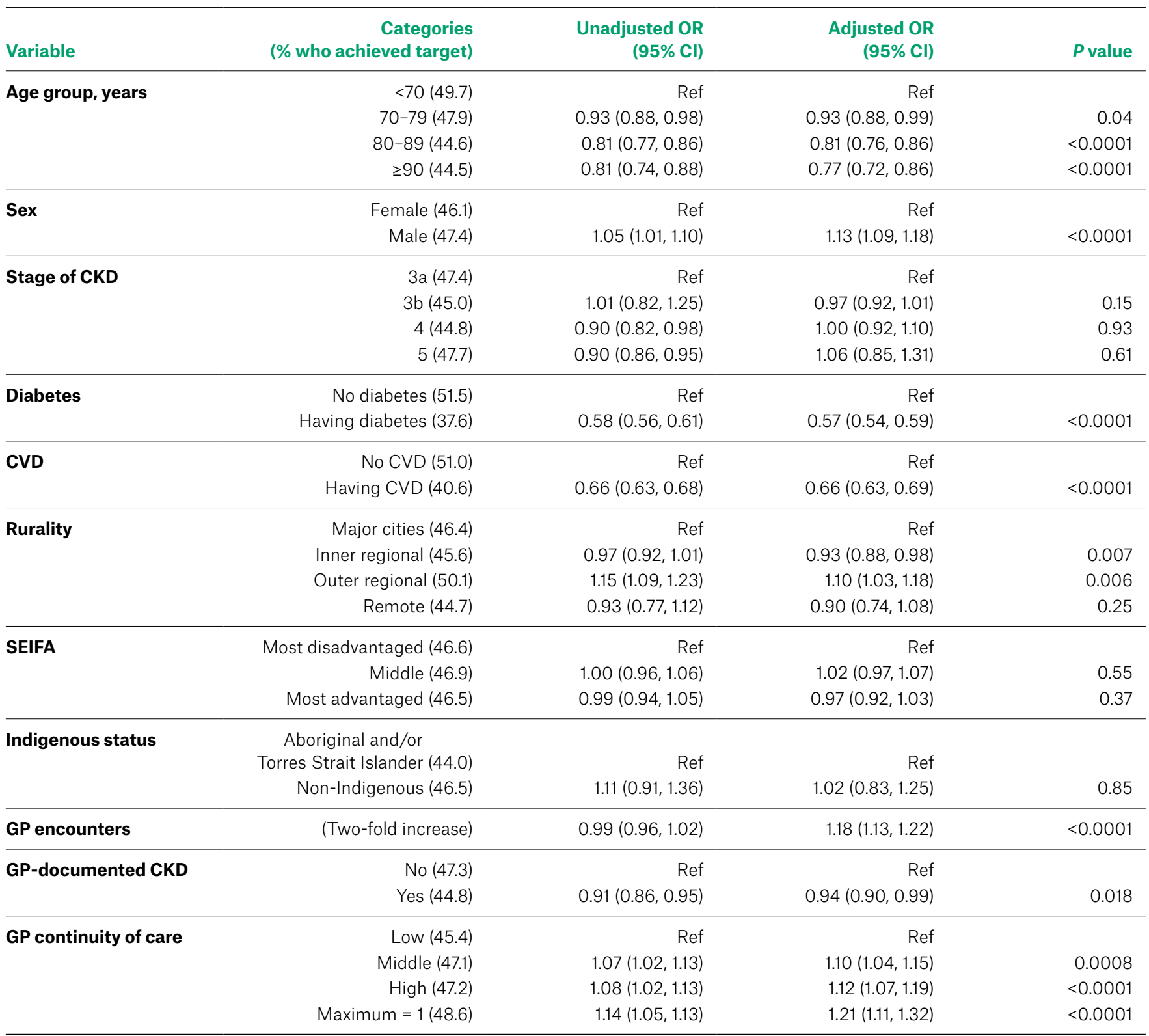

BP, blood pressure; Cl, confidence interval; CKD, chronic kidney disease, CVD, cardiovascular disease; eGFR; estimated glomerular filtration rate; GP, general practitioner; OR, odds ratio; Ref, reference; SEIFA, Socio-Economic Indexes for Areas

10. Perkovic V, Ninomiya T, Arima $H$, et al. Chronic kidney disease, cardiovascular events, and the effects of perindopril-based blood pressure lowering: Data from the PROGRESS study. J Am Soc Nephrol 2007;18(10):2766-72. doi: 10.1681/ ASN.2007020256

11. Kidney Health Australia. Chronic kidney disease (CKD) management in general practice. 2 nd edn. Melbourne: Kidney Health Australia, 2012.

12. National Heart Foundation of Australia and Cardiac Society of Australia and New Zealand. Reducing risk in heart disease: An expert guide to clinical practice for secondary prevention of coronary heart disease. Melbourne: National Heart Foundation of Australia, 2012.

13. Okonofua EC, Simpson KN, Jesri A, Rehman SU, Durkalski VL, Egan BM. Therapeutic inertia is an impediment to achieving the Healthy People 2010 blood pressure control goals. Hypertension 2006;47(3):345-51. doi: 10.1161/01. HYP.0000200702.76436.4b.

14. Guthrie B, Saultz JW, Freeman GK, Haggerty JL. Continuity of care matters. BMJ 2008;337:a867. doi: 10.1136/bmj.a867.
15. Konrad TR, Howard DL, Edwards LJ, Ivanova A, Carey TS. Physician-patient racial concordance, continuity of care, and patterns of care for hypertension. Am J Public Health 2005:95(12):2186-90. doi: 10.2105/ AJPH.2004.046177.

16. Hong JS, Kang HC, Kim J. Continuity of care for elderly patients with diabetes mellitus, hypertension, asthma, and chronic obstructive pulmonary disease in Korea. J Korean Med Sci 2010;25(9):1259-71. doi: 10.3346/ jkms.2010.25.9.1259. 
17. AlHW Australian GP Statistics and Classification Centre. SAND abstract No. 114 from the BEACH program 2007-08: Chronic kidney disease among general practice patients. Sydney: AGPSCC University of Sydney, 2008.

18. Charles J, Britt H, Ng A. Management of cardiovascular conditions in Australian general practice. Aust Fam Physician 2005;34(6):410-11.

19. Medicinelnsight. Medicinelnsight data book version 1.1. Sydney: NPS MedicineWise, updated 2018.

20. Australian Bureau of Statistics. Australian Statistical Geography Standard (ASGS). Canberra: ABS, 2018. Available at www.abs.gov. au/websitedbs/D3310114.nsf/home/Australian +Statistical+Geography+Standard+(ASGS) [Accessed 23 October 2018].

21. Australian Bureau of Statistics (ABS). Census of population and housing: SocioEconomic Indexes for Areas (SEIFA). Cat. no. 2033.0.55.001. Canberra: ABS, 2013. Available at www.ausstats.abs.gov.au/ ausstats/subscriber.nsf/0/22CE DA8038AF7A ODCA257B3B00116E34/\$File/2033.0.55.001\%20 seifa\%202011\%20technical\%20paper.pdf [Accessed 23 October 2018].

22. Medicinelnsight. Medicinelnsight data book version 1.2. Sydney: NPS MedicineWise, 2016.

23. Pollack CE, Hussey PS, Rudin RS, Fox DS, Lai J, Schneider EC. Measuring care continuity: A comparison of claims-based methods. Med Care 2016;54(5):e30-34. doi: 10.1097/ MLR.0000000000000018.

24. Pereira Gray DJ, Sidaway-Lee K, White E, Thorne A, Evans PH. Continuity of care with doctors - A matter of life and death? A systematic review of continuity of care and mortality. BMJ Open 2018;8(6):e021161. doi: 10.1136/bmjopen-2017-021161.

25. Maarsingh OR, Henry Y, van de Ven PM, Deeg DJ. Continuity of care in primary care and association with survival in older people: A 17-year prospective cohort study. Br J Gen Pract 2016;66(649):e531-39. doi: 10.3399/bjgp16X686101.

26. R Core Team. R: A language and environment for statistical computing. Vienna: R Foundation for Statistical Computing, 2017.

27. Williamson JD, Supiano MA, Applegate WB, et al. Intensive vs standard blood pressure control and cardiovascular disease outcomes in adults aged $\geq 75$ years: $A$ randomized clinical trial. JAMA 2016;315(24):2673-82. doi: 10.1001/ jama.2016.7050.

28. Thomas G, Nally J, Pohl MA. Interpreting SPRINT: How low should you go? Cleve Clin J Med 2016;83(3):187-95. doi: 10.3949/ccjm.83a.15175.

29. Razavian M, Heeley EL, Perkovic $V$, et al. Cardiovascular risk management in chronic kidney disease in general practice (the AusHEART study). Nephrol Dial Transplant 2012;27(4):1396-402. doi: 10.1093/ndt/gfr599.

30. Klag MJ, Whelton PK, Randall BL, et al. Blood pressure and end-stage renal disease in men. N Engl J Med 1996;334(1):13-18. doi: 10.1056/ NEJM199601043340103.

31. Tozawa $M$, Iseki $K$, Iseki $C$, Kinjo $K$, Ikemiya $Y$, Takishita S. Blood pressure predicts risk of developing end-stage renal disease in men and women. Hypertension 2003;41(6):1341-45 doi: 10.1161/01.HYP.0000069699.92349.8C.

32. Haggerty JL, Roberge D, Freeman GK, Beaulieu C. Experienced continuity of care when patients see multiple clinicians: A qualitative metasummary. Ann Fam Med 2013;11(3):262-71. doi: 10.1370/ afm.1499.

33. Saultz JW. Defining and measuring interpersonal continuity of care. Ann Fam Med 2003;1(3):134-43. 